\title{
INFLUENCE OF RELATIVE HUMIDITY ON TENSILE AND COMPRESSIVE CREEP OF CONCRETE AMENDED WITH GROUND GRANULATED BLAST-FURNACE SLAG
}

\author{
E. E. Ambrose ${ }^{1, *}$ and J. P. Forth ${ }^{2}$

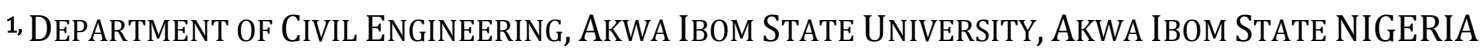 \\ 2, SCHOOL OF CIVIL ENGINEERING, UNIVERSITY OF LEEDS, UNITED KINGDOM \\ E-mail addresses: ${ }^{1}$ edidiongambrose@aksu.edu.ng, ${ }^{2}$ J.P.Forth@leeds.ac.uk
}

\begin{abstract}
This paper presents an experimental study on the influence of ambient relative humidity on tensile creep of plain concrete amended with Ground Granulated Blast-furnace Slag and compares it with its influence on compressive creep. Tensile and compressive creep tests were carried out on concrete specimens of 34.49MPa compressive strength and 0.56 water/binder ratio at 51, 68 and 100\% relative humidity. The results show a linear relationship between compressive creep and relative humidity; this cannot be said about tensile creep. Tensile creep was observed to be more sensitive to change in ambient humidity than compressive creep. Based on equal applied stress, tensile creep was found to be several times higher than compressive creep and the difference was greater in drying creep than in basic creep. On the basis of equal stress/strength ratio, tensile-to-compressive creep ratio was slightly less than 1 for drying creep and much less for basic creep.
\end{abstract}

\section{Keywords: Compressive Creep, Concrete, Relative Humidity, Strain, Strength, Tensile Creep}

\section{INTRODUCTION}

For decades - if not centuries - concrete has been used all over the world in different forms of constructions as a consequence of its versatility and sustainability credentials. Concrete is basically a series of aggregates bonded together with an adhesive or binder which is usually hardened cement paste formed by the hydration of cement. Two forms of deformations take place when concrete is subjected to constant or cyclic loading. First is the instantaneous deformation which is basically the elastic strain as a result of the external load and secondly there is the time-dependent deformation which is a combination of shrinkage and creep; both occur simultaneously and increase with time but at a diminishing rate. While creep is as a result of sustained stress, shrinkage is due to hygrometric conditions [1]. According to Neville et al [2], a typical concrete element creeps up to twice its initial strain after a year of loading. Concrete creep can occur in tension and also in compression. The properties of tensile and compressive creep are usually considered to be similar $[3,4]$ but several researchers have revealed that their mechanisms are quite different [5]. Several mechanisms have been proposed for creep. Each of these theories explains a number of observations which is in line with certain experimental results but none can give a complete explanation to all the observed behaviours. Many researchers have accepted that seepage and viscous flow are the major contributions to the creep mechanism. Domone [6] suggested that the seepage mechanism produces reversible creep while viscous flow produces irreversible deformation and that these two deformations are present in tensile and compressive creep. Still on seepage, Domone observed that while compressive creep involves the expulsion of gel water (moisture) from the gel pores to the capillary pores, tensile creep involves the migration of moisture away from the capillary pores. Illston [7] had suggested that creep - both in compression and in tension - is due to viscous flow and delayed elasticity. Reinhardt and Rinder [8] observed a new phenomenon in tensile creep tests with high strength concrete. That is: the hydration rate and shrinkage in loaded specimens were more than in non-loaded specimens. It was then suggested that this might be an explanation to Powers' creep theory which holds that moisture movement (seepage) is the fundamental creep mechanism [8]. After a series of investigations using acoustic emission, Rossi et. al. $[4,9]$ reported that basic creep of concrete 
is proportional to the number of microcracks created within the concrete element. It was further added that microcrack initiation causes hygral imbalance within concrete elements, which in turn causes moisture movement from capillary pores to microcrack pores leading to self-drying of the capillaries and, hence, additional shrinkage. It was therefore proposed that microcrack initiation is the origin of basic creep $[4,9]$. As the puzzle behind the actual mechanism of tensile and compressive creep of concrete is still unresolved, it is, however, generally accepted that they take place in the hydrated cement paste and are caused by the movement of absorbed water within and between the capillary pores and the cement gel pores in the microstructure of concrete $[2,6,7,10]$.

There is currently limited literature comparing experimental tensile and compressive creep data for the same test conditions and the few available are not consistent in their findings [3]. This is partly due to the difficulty in carrying out tensile tests on cement-based materials $[3,5,11]$ as a result of their poor tensile strain capacity and brittle nature. Hence, investigations into concrete creep are often limited to the concrete's behaviour in compression. However, tensile creep reduces tension stiffening in reinforced concrete members in the long term and a combination of tensile and compressive creep increases curvature with time in reinforced concrete members. These two mechanisms according to Forth [12] enhance long term deflection. Creep is also the key mechanical property that determines the stress build-up and related cracks in restrained structural members. Hence, a good understanding of creep both in tension and in compression will not only help in achieving reliable crack assessment methods [3] but also in predicting long term deflection of concrete members. Moreover, a detailed knowledge of tensile creep aids in the prediction of tensile stresses in prestressed concrete beams and in the design of water retaining structures [13].

This paper presents an experimental study on the influence of ambient relative humidity on tensile creep of plain concrete amended with Ground Granulated Blast-furnace Slag and compares it with its influence on compressive creep.

\section{MATERIALS AND METHODS}

\subsection{Materials}

\subsubsection{Binder}

The binder used for this study was cement, with $40 \%$ replacement with Ground Granulated Blast-furnace Slag (GGBS). The cement used was Ordinary Portland
Cement (strength class 52.5N) manufactured by Hanson Cement Group (UK) in compliance with BS EN 197-1:2011 requirement [14] and the GGBS used was in compliance with BS EN 15167-1:2006 requirement [15].

\subsubsection{Aggregate}

The selection of aggregates used for the casting of the specimens was in compliance with BS EN 206:2013 [16] and BS EN 12620:2002+A1:2008 [17] and the aggregates were supplied by Lafarge Tarmac Limited (UK). Silica sand and gravel from Finningley Quarry (UK) with maximum size of $20 \mathrm{~mm}$ and uniformity coefficient of 3, was used as coarse aggregate while sand and gravel from Scorton (UK) with uniformity coefficient of 11 was used as fine aggregate.

\subsection{Concrete Production}

The mix composition for the concrete mix was as presented in Table 1. A water-binder ratio of 0.56 was used for the mix. The materials were mixed using a mechanical concrete mixer and compaction was obtained using a vibrating table. To eliminate the influence of the intrinsic variation in properties of concrete on the different specimens, a single batch of concrete was used for all the specimens and the batching was by weight. Concrete cubes for compressive strength tests, concrete cylinders for split cylinder (indirect tensile strength) tests, concrete prisms for compressive creep tests and concrete bobbins for direct tensile strength tests and tensile creep tests; were prepared.

Table 1: Mix composition

\begin{tabular}{ll}
\hline Material & $\begin{array}{l}\text { Dry Batch } \\
\text { Weight }\left(\mathrm{kg} / \mathrm{m}^{3}\right)\end{array}$ \\
\hline $\begin{array}{l}\text { Ordinary Portland Cement } \\
\text { (CEM 1 52,5 N) }\end{array}$ & 198 \\
$\begin{array}{l}\text { GGBS } \\
\text { Fine aggregate (sand and }\end{array}$ & 132 \\
$\begin{array}{l}\text { gravel) } \\
\text { Coarse aggregate (silica sand } \\
\text { and gravel) }\end{array}$ & 736 \\
Water $(\mathrm{w} / \mathrm{c}=0.56)$ & 1104 \\
\hline
\end{tabular}

\subsection{Test Methods}

\subsubsection{Cube Compressive Strength Tests}

The test specimens for the compressive strength tests were $100 \times 100 \times 100 \mathrm{~mm}$ concrete cubes and the testing were carried out in accordance with BS EN 123903:2009 [18]. The tests were carried out for the 14-day and 28-day strengths and 2 samples were tested for 
each strength and the average was taken as the strength for each. The results are presented in Table 2 .

\subsubsection{Direct Tensile Strength Test}

The direct tensile strength tests were carried out using bobbins of $75 \mathrm{~mm}$ diameter as test specimens. The tests were carried out for the 14-day and 28-day direct tensile strengths and the obtained results are presented in Table 2.

\subsubsection{Split Cylinder Test}

The split cylinder (indirect tensile strength) tests were carried out in compliance with BS EN 12390-6:2000 [19]. The test specimens were $150 \mathrm{~mm}$ diameter concrete cylinders with $300 \mathrm{~mm}$ heights. The tests were carried out for the 14-day and 28-day strengths and the results are presented in Table 2.

\subsubsection{Compressive Creep Tests and Measurements}

Compressive creep was measured on concrete prisms (Figure 1a) using the test set-up shown in Figure 2. The prisms were $200 \mathrm{~mm} \times 75 \mathrm{~mm} \times 75 \mathrm{~mm}$ each. Three separate rigs with two specimens each were used for the three compressive creep investigations at 51, 68 and $100 \% \mathrm{RH}$. The tests were carried out in controlled environment creep rooms. The 51\% and 68\% RH tests were carried out in two separate creep rooms, while for both compressive and tensile creep tests, the $100 \% \mathrm{RH}$ was obtained by sealing the specimens with double layers of self-adhesive aluminum foil. The sealing was to prevent any moisture exchange with the environment and is a well-established practice in creep and shrinkage experiments $[4,11,12]$. All compressive creep specimens were cured in a fog room at $99 \% \mathrm{RH}$ and $20{ }^{\circ} \mathrm{C}$ until the beginning of the tests in the controlled environment creep rooms. To eliminate the influence of stress level and age of loading, a uniform stress of $12.26 \mathrm{MPa}$ was used for the three compressive creep tests and the specimens were all loaded at the age of 14 days. The applied stress represents a stress level of $36 \%$ of the compressive strength of the concrete specimens at the age of loading. Creep measurements were carried out using Demountable Mechanical Gauges (DEMEC) in the usual way [20]. For both compressive and tensile creep investigations, shrinkage was also investigated on separate concrete prisms and bobbins. These specimens were unloaded and stored in the same respective $\mathrm{RH}$, such that creep was obtained as the difference between the total timedependent strain and shrinkage strain. For the purpose of equal stress comparison with tensile creep, the specific creep (creep per unit stress) values in compression were also computed.

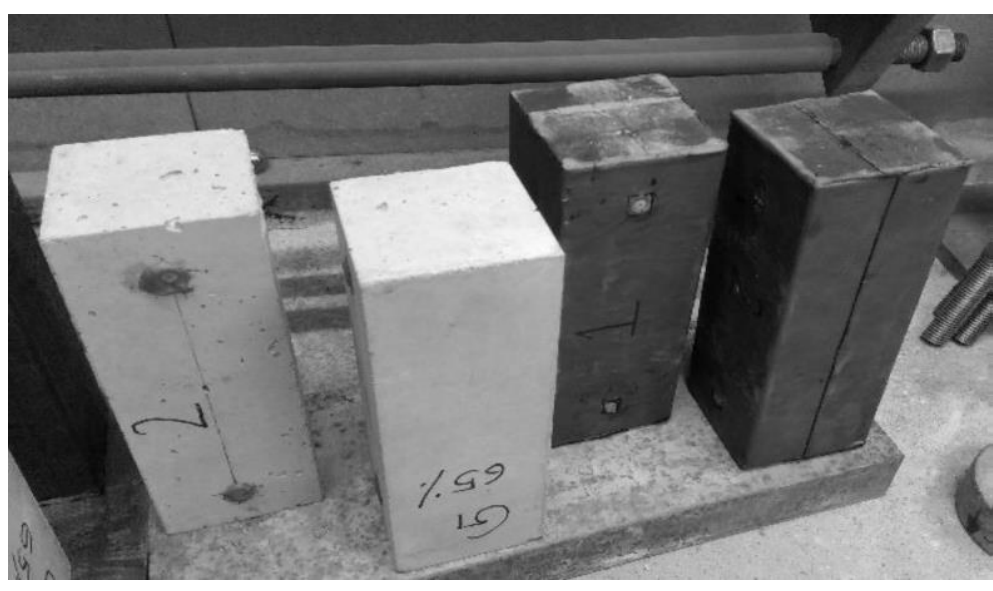

a) Concrete prisms

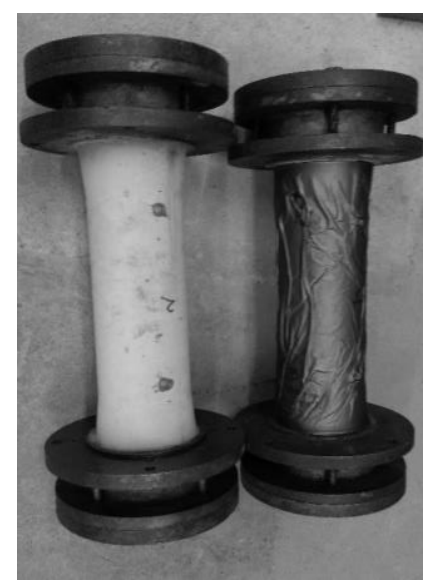

b) Concrete bobbins

Figure 1: Creep tests specimens (sealed and unsealed)

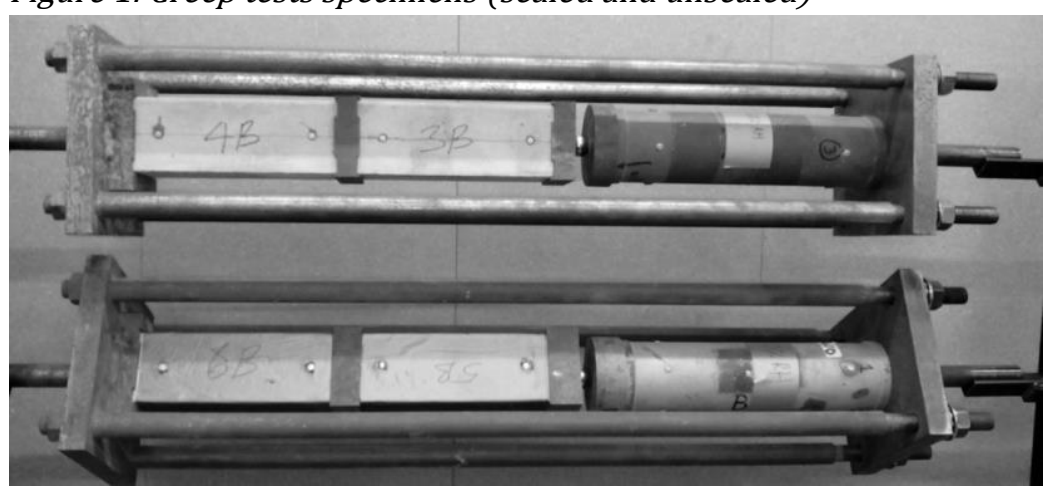

Figure 2: Compressive creep rigs showing sealed and unsealed specimens 


\subsubsection{Tensile Creep Tests and Measurements}

All tensile creep tests were carried out on concrete bobbins with $75 \mathrm{~mm}$ diameter (Figure 1b), using the test set-up shown in Figure 3. Three separate rigs were used for three tensile creep investigations carried out under the same environmental conditions as those of the compressive creep tests (that is: 51,68 and $100 \%$ $\mathrm{RH})$. Concrete bobbins for the tests were left in their moulds and not in the fog room, until the beginning of the tests in the controlled environment creep rooms. The specimens were loaded on the $14^{\text {th }}$ day after casting and a stress of $1 \mathrm{MPa}$ corresponding to a stress/strength ratio of 0.34 , was used for all the tensile creep tests. This low applied stress was to avoid instantaneous failure of the specimens due to concrete's low tensile strain capacity

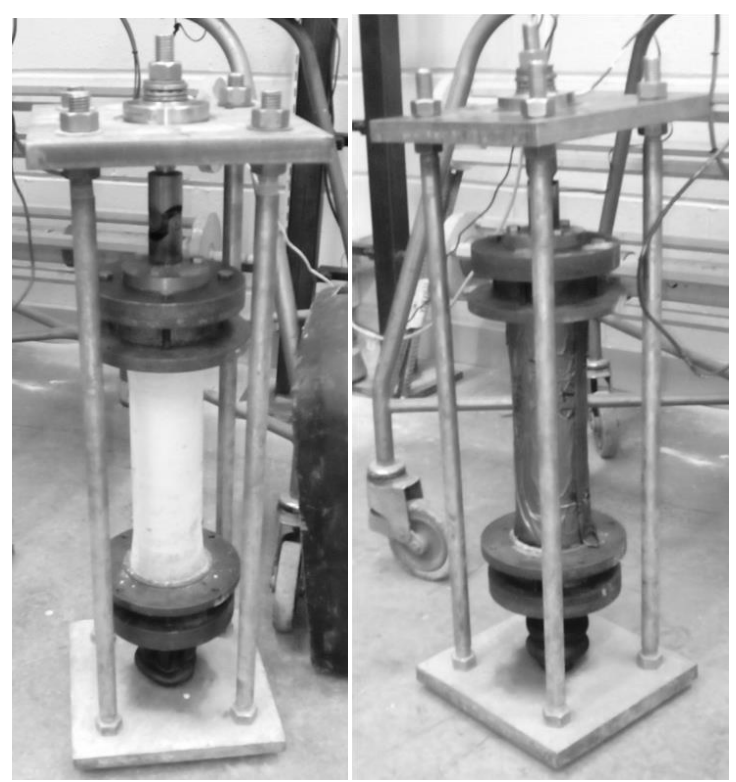

Figure 3: Tensile creep rigs showing sealed and unsealed specimens

\section{RESULTS AND DISCUSSIONS}

\subsection{Relative Humidity (RH) Variation}

The daily average $\mathrm{RH}$ variation for the two creep laboratories are presented in Figure 4. It is obvious that the $\mathrm{RH}$ for the duration of the creep tests was not constant. The average RH for Laboratory 1 throughout the duration was $51 \%$ while for Laboratory 2 , it was $68 \%$. Of course these variations have a great influence on the drying creep results. Literature reveals that there is a significant influence of cyclic variation of ambient humidity on creep values. Test specimens subjected to cyclic humidity may creep as much as twice the creep of specimens subjected to a constant humidity equal to the average cyclic humidity [21]. This effect does not affect shrinkage values of the control specimens [2], rather it increases the magnitude of the total deformation of the loaded specimens by enhancing their induced shrinkage and hence influence the reported drying creep values as there will be more microcracks in loaded specimens. However, although this variation was not intentional, it is a situation that frequently exists in real structures.

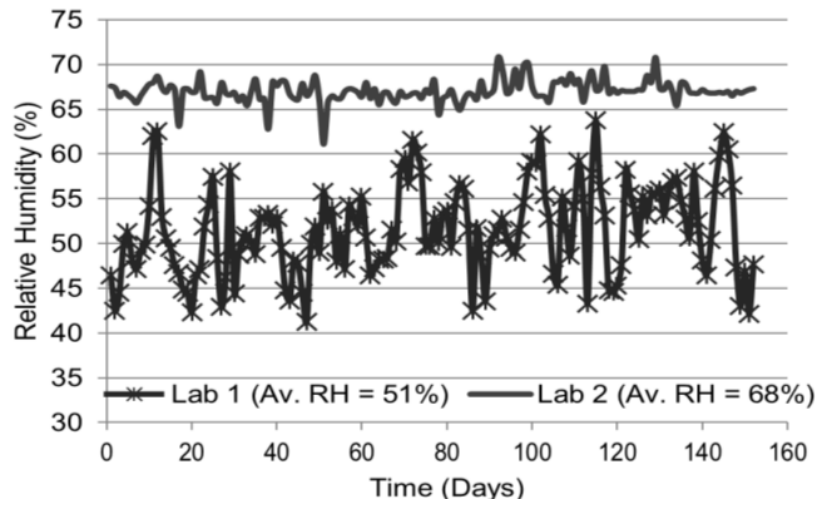

Figure 4: Average Daily RH variation for the 2 creep Laboratories

\subsection{Compressive Creep at Varying $R H$}

The results of the three compressive creep tests are presented in Figure 5. The concrete specimens' strength test results are shown in Table 2. Creep results in this study were computed based on the usual assumption that creep and shrinkage are additive and independent of each other - the principle of superposition. Hence, creep values were obtained by finding the difference between the total timedependent strain and shrinkage of similar specimens unloaded and stored under the same conditions for the same period (controlled specimens). As expected, the plots of the compressive creep strains all show the usual increase with time but at a decreasing rate. This is consistent with the available creep data in the literature. The influence of ambient humidity on compressive creep of concrete is explicitly demonstrated in Figure 6. Specimens loaded at a lower RH creep more than those loaded at a higher RH. The sealed specimens $(100 \% \mathrm{RH})$ recorded the lowest creep magnitudes because under such conditions, no moisture exchange takes place between the specimens and the surrounding environment. Hence, there is usually no drying of such specimens. The moisture content within the specimens also affects creep and this is controlled by the extent of drying of the specimen which is also determined by the humidity of the ambient medium. All these point to the fact that moisture movement - seepage - is a fundamental mechanism of creep. 
Table 2: Results of concrete strength tests

\begin{tabular}{llll}
\hline Age(Days) & Cube compressive strength test (MPa) & Split cylinder test (MPa) & Direct tensile strength test (MPa) \\
\hline 14 & 34.49 & 2.96 & 2.04 \\
28 & 40.71 & 3.25 & 2.24 \\
\hline
\end{tabular}

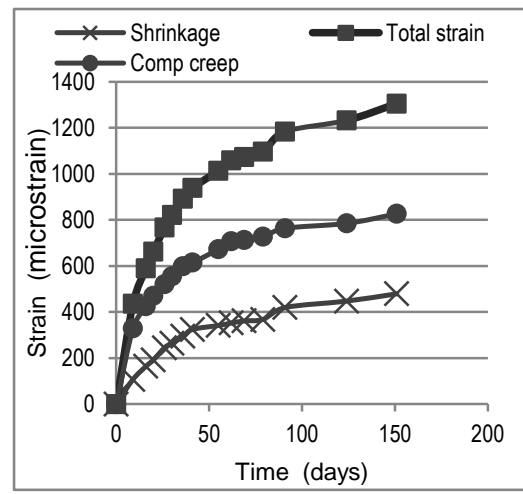

a) $51 \% \mathrm{RH}$

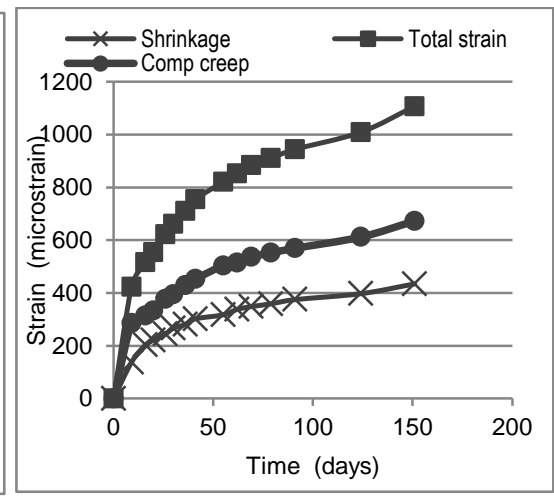

b) $68 \% \mathrm{RH}$

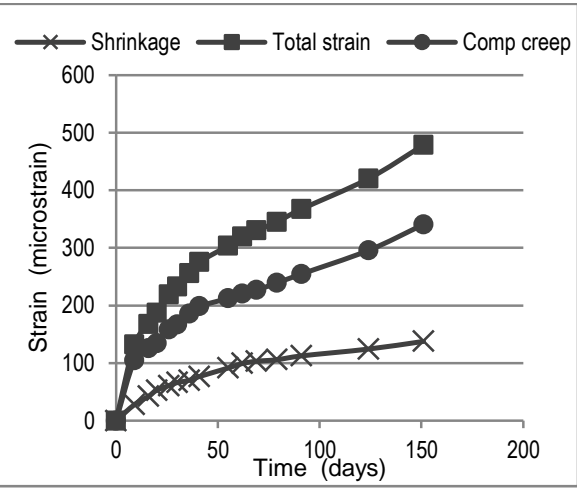

c) $100 \% \mathrm{RH}$

Figure 5: Total strain, shrinkage strain and compressive creep at different $R H$
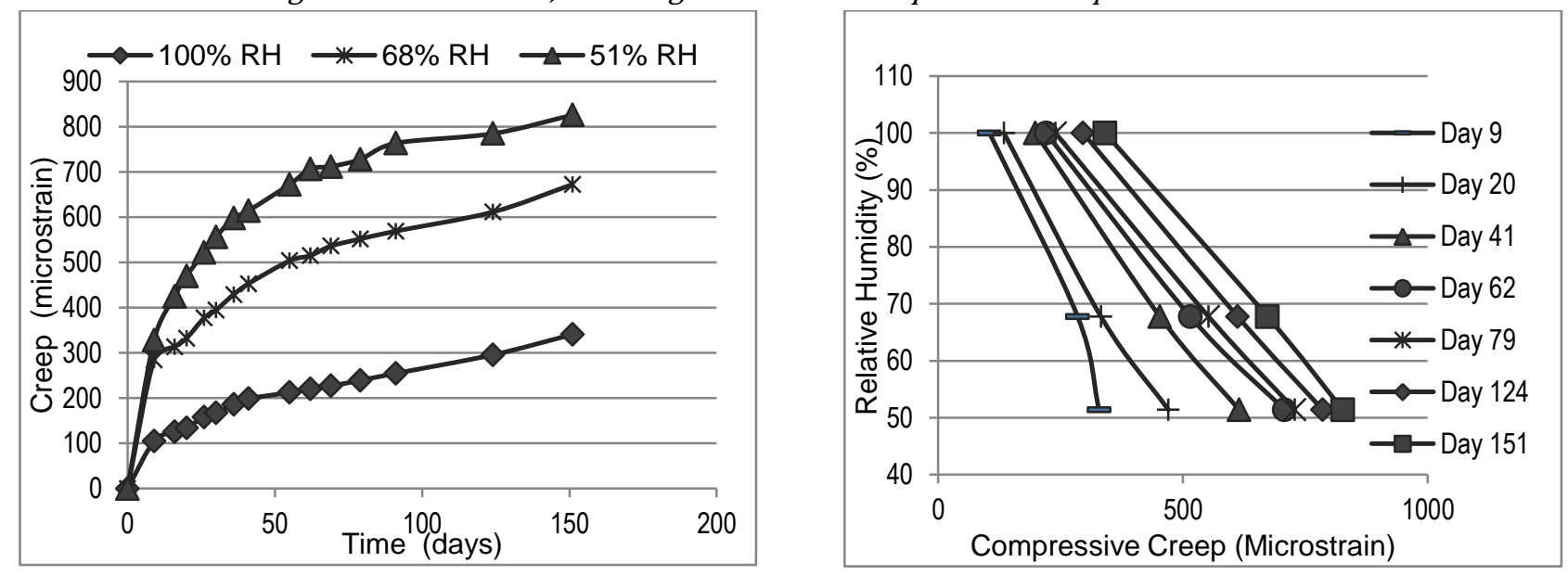

Figure 6: Compressive creep at different $R H$

Figure 7: Relationship between compressive Creep and RH

Figure 7 shows an inverse relationship between compressive creep and $\mathrm{RH}$ at different stages throughout the experiment. This relationship is linear or approximately linear, depending on the time under load. This approximately linear relationship at early ages is reported elsewhere [2].

\subsection{Tensile Creep at Varying $R H$}

As presented in Figure 8, tensile creep values increased with time but with the usual decreasing rate. Tensile creep values were mostly influenced by their respective shrinkage strain values as the latter were usually of several magnitudes greater than their respective total strain values. The increase of creep with time can be explained by the progressive microdifussion of moisture from the gel pores to the capillary pores while the decrease in creep rate is due to the increase in hydration of the cement paste with age and hence, the corresponding increase in strength
[22]. Figure 9 shows the results of the three tensile creep tests at different RH. An inverse relationship between creep and RH was again present. Tensile creep values recorded from the 51\% RH test were higher than those of the tests carried out at $68 \%$ and $100 \%$ RH. The test with the sealed specimen recorded the lowest values of tensile creep. This is because the specimens were unaffected by the ambient $\mathrm{RH}$ and hence there was no moisture exchange with the ambient medium. The increase in creep of the unsealed specimens with decreasing $\mathrm{RH}$ is due to the drying creep which is a function of the ambient humidity. Figure 10 shows an inverse relationship between tensile creep and RH. The relationship is less linear than that seen for compression creep. The non-linearity is more pronounced at the early stage of loading; and as the age of loading increases, the relationship tends towards linear. 


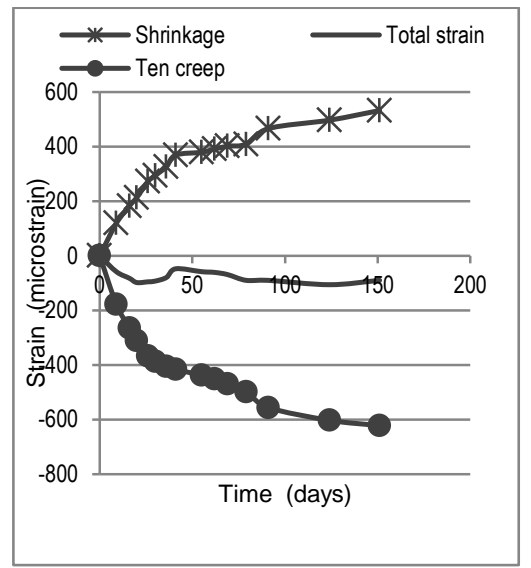

a) $51 \% \mathrm{RH}$

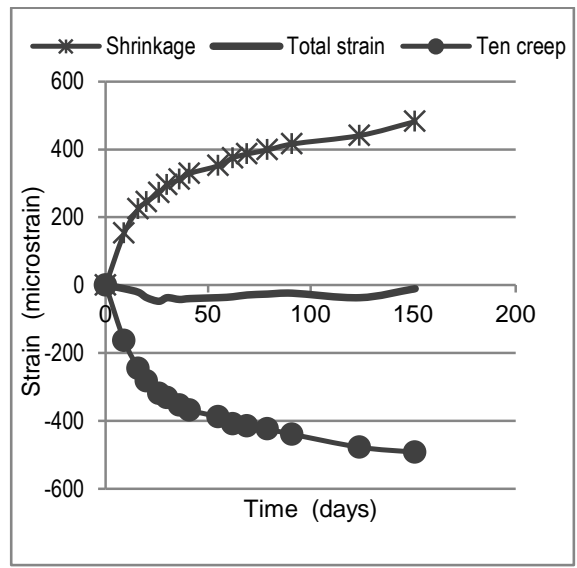

b) $68 \% \mathrm{RH}$

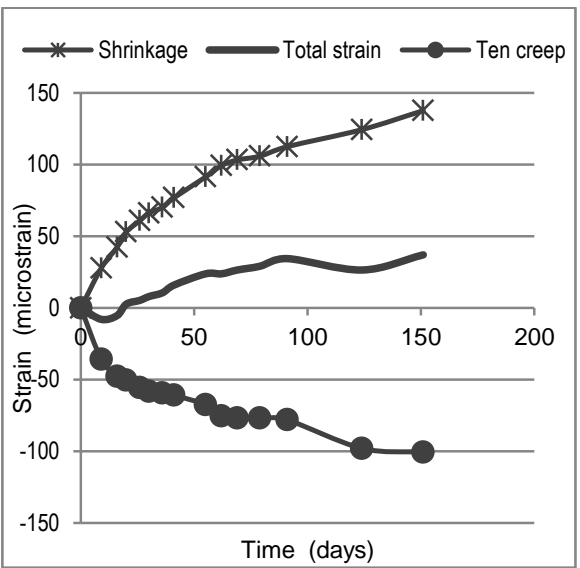

c) $100 \% \mathrm{RH}$

Figure 8: Total strain, shrinkage strain and Tensile creep at different $R H$

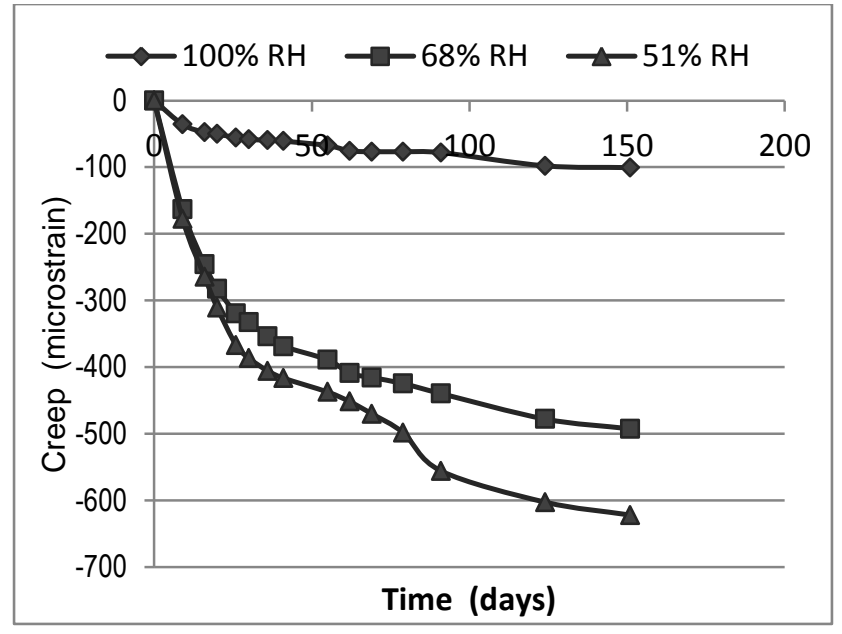

Figure 9: Tensile creep at different $R H$

The following should however be noted. In using the superposition principle of creep and shrinkage to derive creep values it is important to measure shrinkage of the control samples accurately as it plays a significant role in determining creep values. As can be seen in Figure 8, for tensile creep tests, shrinkage values of the control specimens were of several magnitudes higher than their corresponding total strain values which at most points were negative. Meanwhile, there is an argument that shrinkage of a loaded specimen could be greater than that of a control sample [11, 20] and as such, subtracting the shrinkage strain of a controlled specimen from the total deformation of a loaded sample to get creep values is only a convenient simplification which is not exactly true in reality. This argument is anchored on the existence of load-induced shrinkage and microcracking effect on loaded samples and this is particularly significant in tension samples which have more microcracks and hence more induced shrinkage than compression samples. It is well established that concrete cracks even with loading below its load

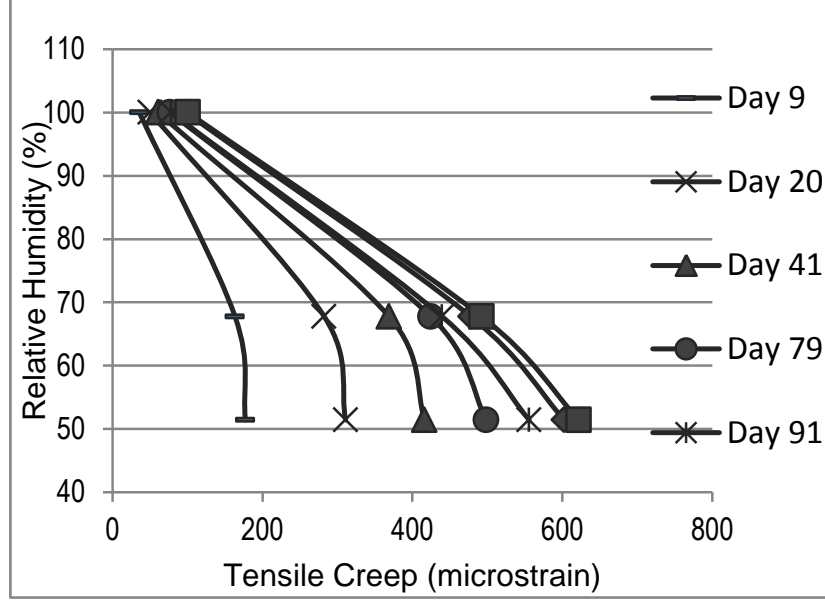

Figure 10: Relationship between tensile creep and $R H$

bearing capacity and according to Rossi et al. [4] there is a direct relationship between stress level (both in tension and compression) in concrete and the density of microcracks. For a loaded specimen, these loadinduced microcracks which localize at the cement paste-aggregate interface [23] combined with the preexisting cracks created by the restrained shrinkage of the cement paste by the coarse aggregate [24], could cause internal moisture gradients [11] which develop additional internal stresses within the concrete and therefore amplify shrinkage of the loaded specimen. However, the difficulty is in quantifying the magnitude of this additional shrinkage and decoupling it from the actual stress-independent shrinkage.

\subsection{Basic and Drying Creep}

The magnitude of creep is influenced by the intrinsic $\mathrm{RH}$ of the concrete itself (which is determined by the amount of moisture within the microstructure of the concrete) and the RH of the ambient medium. Whether or not there is moisture exchange between these two medium differentiates between basic creep and drying 
creep. Basic creep takes place when the concrete is in hygral equilibrium with the ambient medium meaning there is no moisture loss from the concrete. However, creep is hugely affected by the drying of concrete under load. Hence drying concrete (concrete which losses moisture to its environment) creeps more than concrete that remains wet or dry. At $50 \% \mathrm{RH}$, concrete may creep two to three times its value at $100 \%$ [2]. This is confirmed in this investigation as shown in Figures 6 and 9 where the magnitudes of creep - both in tension and in compression - are shown to be inversely proportional to RH. The increase in creep with decreasing $\mathrm{RH}$ is due to the additional strain caused by simultaneous drying and loading. This excess deformation referred to as drying-induced creep (or simply drying creep) was first reported by Pickett [10] when the deformation of a specimen subjected to simultaneous drying and sustained loading was observed to be greater than the sum of the drying shrinkage from a similar specimen with no loading and the creep deformation of another similar specimen which does not dry (sealed specimen) but is loaded. It therefore appears that drying creep is still linked with load-induced shrinkage caused by microcracking effect and the drying gradient developed by the nonuniformity of shrinkage within the concrete specimen thereby subjecting the surface layer to tension which leads to further microcracking (tensile cracking).

\subsection{Tensile/Compressive Creep Ratio}

The result of comparing tensile and compressive creep data depends on the method of comparison. Some literatures compare the two properties based on equal magnitude of applied stresses, while others carry out the comparison based on an equal stress/strength ratios and each of these methods has its limitations.

\subsubsection{Based on Equal Applied Stress}

On the basis of equal applied stress - in the case of this investigation, specific creep as shown in Figure 11 -

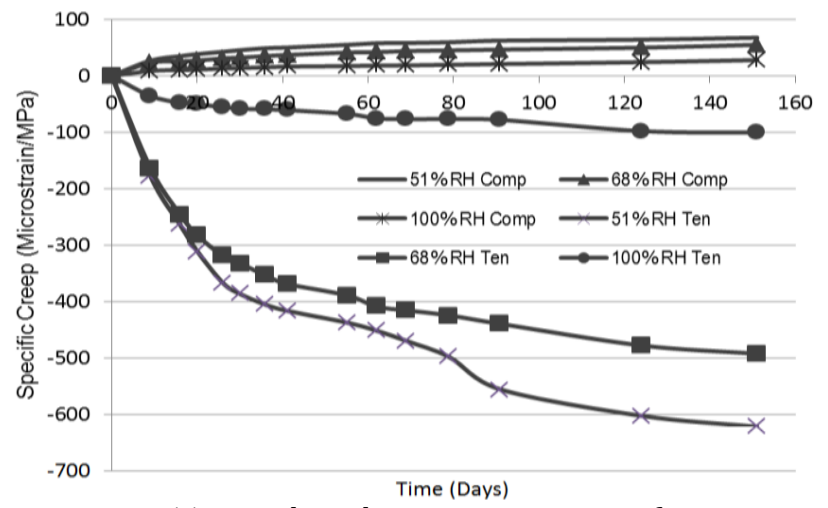

Figure 11: Tensile and compressive creep of concrete specimens tested at different $R H$ based on equal stress level creep is several times higher in tension than in compression at the three observed RH. It is therefore reasonable to suggest that on this basis, the tensile-tocompressive creep ratio is greater than 1 . This is in line with the findings of Brooks and Neville [25]. From Figure 11, this ratio is observed to be higher for drying creep than for basic creep, which is an indication that the effect of ambient humidity is higher in tensile creep than in compressive creep. This is however not surprising considering the existence of shrinkageinduced creep in drying specimens. This could also hold the explanation for the non-linearity in the relationship between tensile creep and ambient humidity (Figure 10) as against the linear relationship in compressive creep (Figure 7). However, it must be remembered and noted that the values for compressive creep in this comparison were not actually from tests carried out at $1 \mathrm{MPa}$ (as in the case of tensile creep), rather they were specific creep values derived from tests carried out at 12.26MPa. Specific creep values have been reported to be less than actual creep values obtained from tests carried out at $1 \mathrm{MPa}$ [12]. Hence, the tensile-tocompressive creep ratio derived is not truly definitive, rather indicative of the difference between the two properties.

\subsubsection{Based on Equal Stress/strength Ratio}

With equal stress/strength ratios, the applied stress in the compressive tests are usually several times higher than those of the tensile tests, because of the low tensile strength of concrete in comparison with its compressive strength. For this investigation, the applied stress for comparison on the basis of equal stress/strength ratio was $1 \mathrm{MPa}$ and $12.26 \mathrm{MPa}$ for the tensile and compressive creep test, respectively. The result of the comparison (Figure 12) shows that the tensile-to-compressive creep ratio is slightly less than 1 for drying creep and much less for basic creep.

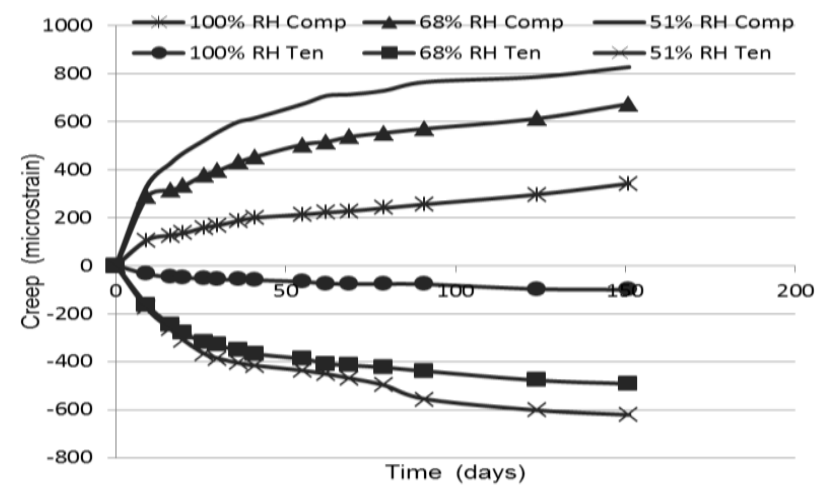

Figure 12: Tensile and compressive creep of concrete specimens tested at different $R H$ based on equal stress/strength ratio

Vol. 37, No. 1, January 2018 
However, the following should be noted; there are more microcracks in the tension samples and so there is more induced shrinkage in the loaded tension specimens. If the shrinkage in the unloaded samples underestimates the shrinkage in the loaded samples because of the presence of induced shrinkage in the loaded samples, then the compressive creep in the loaded compression samples is overestimated while the tensile creep in the loaded tension samples is underestimated. The upshot is that for equal stress/strength ratios, the ratio of tensile-tocompressive creep might be approximately 1 or even greater than 1.

\section{CONCLUSION}

The aim of this experimental work was to investigate the influence of ambient humidity on tensile creep of plain concrete amended with GGBS and to compare it with its influence on compressive creep of plain concrete. This was achieved experimentally by monitoring the tensile and compressive creep of concrete specimens (prism for compressive creep and bobbins for tensile creep) under 51, 68 and 100\% RH. From the results, the following can be deduced.

- Both compressive and tensile creep of plain concrete increase with decrease in $\mathrm{RH}$ due to drying creep. This inverse relationship has been confirmed to be linear in compression as reported in several previous works. However, there are indications that it might not be linear at early stage of loading.

- The study shows that the relationship between tensile creep and RH is not linear as previously assumed. However, the non-linearity reduced as the experiment progressed; hence it is possible that it might be linear in the very long term.

- Based on equal applied stress, tensile creep is several times higher than compressive creep. The tensile-to-compressive creep ratio is higher in drying creep than in basic creep indicating that the indirect influence of ambient humidity is greater on tensile creep than in compressive creep.

- On the basis of equal stress/strength ratio, tensileto-compressive creep ratio is slightly less than 1 for drying creep and much less for basic creep. However, there are strong reasons to suggest that this ratio might actually be approximately 1 or even greater.

\section{REFERENCES}

[1] Tamtsia, B. T. and Beaudoin, J. J. "Basic Creep of Hardened Cement Paste: A Re-examination of the Role of Water", Cement and Concrete Research, Vol. 30, pp 1465-1475, 2000.

[2] Neville, A. M. et al. Creep of Plain and Structural Concrete, Construction Press, Paperback, London, 1983.

[3] Ji, G. M. "Tensile and Compressive Creep Deformations of Hardening Concrete Containing Mineral Additive", Materials and Structures, Vol. 46, pp 1167-1182, 2013.

[4] Rossi, P. et al. "Comparison of Concrete Creep in Tension and in Compression: Influence of Concrete Age at Loading and Drying Conditions", Cement and Concrete Research, Vol. 51, pp 78-84, 2013.

[5] Ranaivomanana, N. et al. "Tensile, Compressive and Flexural Basic Creep of Concrete at Different Stress Levels", Cement and Concrete Research, Vol. 52, pp 1-10, 2013.

[6] Domone, P. L. "Uniaxial Tensile Creep and Failure of Concrete", Magazine of Concrete Research, Vol 26, Number 88, pp 144-152, 1974.

[7] Illston, J. M. "The Creep of Concrete under Uniaxial Tension", Magazine of Concrete Research, Vol. 17, Number 51, pp 77-84, 1965.

[8] Reinhardt, H. and Rinder, T. "Tensile Creep of High Strength Concrete", Journal of Advance Concrete Technology, Vol. 4, Number 2, pp 277-283, 2006.

[9] Rossi, P. et al. "Basic Creep Behaviour of Concretes Investigation of the Physical Mechanisms by Using Acoustic Emission", Cement and Concrete Research, Vol. 42, pp. 61-73, 2012.

[10] Bazant, Z. P. and Chern, J. C. "Concrete Creep at Variable Humidity: Constitutive Law and Mechanism", Materiaux et al Constructions, Vol. 18, Number 1, pp 1-20, 1985.

[11] Ranaivomanana, N. et al. "Basic Creep of Concrete under Compression, Tension and Bending", Construction and Building Materials, Vol. 38, pp 173-180, 2013.

[12] Forth J. P. "Predicting the Tensile Creep of Concrete", Cement and Concrete Composites, Vol. $55, \mathrm{pp}$

70-80, 2015.

[13] Østergaard, L. et al. "Tensile Basic Creep of Earlyage Concrete under Constant Load", Cement and Concrete Research, Vol. 31, 2001, pp 1895-1899.

[14] BS EN 197 Part 1. Cement - Specification and Conformity Criteria, British Standard Institute, Brussels, 2011. 
[15] BS EN 15167 Part 1. Ground Granulated Blastfurnace Slag for Use in Concrete, Mortar and Grout - definitions, Specifications and Conformity Criteria, British Standard Institute, Brussels, 2006.

[16] BS EN 206. Concrete - Specification, Performance, Production and Conformity, British Standard Institute, Brussels, 2013.

[17] BS EN 12620. Aggregate for Concrete, British Standard Institute, Brussels, 2002.

[18] BS EN 12390 Part 3. Compressive Strength of Test Specimens, British Standard Institute, Brussels, 2009.

[19] BS EN 12390 Part 6. Tensile Splitting Strength of Test Specimens, British Standard Institute, Brussels, 2000.

[20] Neville, A. M. Properties of Concrete (5th ed.), Pearson Education, Paperback, London, 2011.

[21] Bazant, Z. P. and Wang, T. "Practical Prediction of Cyclic Humidity Effect on Creep and Shrinkage of
Concrete", Materiaux et Constructions, Vol. 18, Number 4, pp. 247-252, 1985.

[22] Al-Kabaisy, M. A. and Young, A. G. "Failure of Concrete under Sustained Tension", Magazine of Concrete Research", Vol. 27, Number 92, pp. 171178, 1975.

[23] Brooks, J. J. and Neville, A. M. "Comparison of Creep, Elasticity and Strength of Concrete in Tension and in Compression", Magazine of Concrete Research, Vol.29, pp.131-141, 1977.

[24] Olonade, K. A., Kadin, M. B. and Aderemi, P. 0. "Performance of steel slag as fine aggregate in structural concrete", Nigerian Journal of Technology, Vol. 34, No. 3, pp. 452 - 458, 2015.

[25] Falade, F., Ukponu, B., and Ugbaja, J. O., "Influence of superplasticizer and varying aggregate size on the drying shrinkage and compressive strength of laterised concrete", Nigerian Journal of Technology, Vol. 36, No. 3. Pp. 734 - 739, 2017. 